\title{
The Search for MET or a MET Homolog Expressed by Dugesia Dorotocephala
}

\author{
Jonathan F. Blaize ${ }^{1}$ and Sherry J. Browne ${ }^{1}$ \\ ${ }^{1}$ Wagner College, Department of Biological Sciences, New York, USA.
}

The mechanisms controlling morphallactic regeneration are among the most complex and well studied of all biological processes [1]. Since the $18^{\text {th }}$ century planaria have severed as a model organism for the study of regeneration due to their immense developmental plasticity [3], simple body plan and relative abundance [1]. In our investigation, fresh water brown planaria (Dugesia dorotocephala) will be examined $i)$ to determine if this species of Platyhelminthes express MET or a MET homolog, ii) the extent of HGF/MET influence during re-epithelialization following planarian bisection, iii) and whether intracellular signals responsible for planarian recovery are consistent with those activated by human hepatocytes following trauma.

Regeneration of lost or injured planarian tissue is controlled by a litany of molecular cues that initiate prompt cytoskeletal reorganization, re-epithelialization and recruitment of neoblasts to the wound site $[1-5,12,13]$. While few multi-cellular eukaryotes share the restorative fidelity displayed by planaria, nearly all members of the animal kingdom rely upon epithelialization to reduce the likelihood of infection, minimize water loss/maintain tonicity and initiate recovery immediately following severe trauma [1]. Unsurprisingly, many of the cellular hallmarks that facilitate reinstitution of epithelial continuity are conserved between species, despite massive variability within the repair scheme thereafter [1]. A key contributor to this process in mammalian wound healing is the peptide ligand hepatocyte growth factor (HGF) and the receptor tyrosine kinase to which it binds- mesenchymal epithelial transition factor (MET).

HGF has been thoroughly investigated since its discovery and partial characterization in 1984 [6-8]. While initially described as a unique mitogen that participates in liver regeneration, it is now well accepted that the HGF-MET pathway contributes significantly to cellular proliferation, migration and morphogenesis in many organ systems [4,7-11,14]. Considerable evidence illustrates the importance of HGF-MET signaling in development and cellular injury recovery; for example, abolishment of MET expression profoundly effects tissue remodeling and composition of hepatic stem cells and prevents re-epithelialization in epidermal wounds by keratinocytes [2,6]. Two downstream targets of MET activation, phosphoinositide 3-kinase (PI3K) and the family of signal transducers and transcribers (STAT3) are specifically required for re-epithelializationand subsequent regeneration of planarian bodies [5, 11]; interestingly, there has been no investigation of MET or MET homolog expression in planaria. We suspect that MET or a MET-like protein plays a role in planarian regeneration, as it is a key mediator of cellular survival, growth and differentiation.

To determine whether D. dorotocephala express MET, standard immunohistochemical techniques were employed. 10 planarian samples were stripped of their mucosal lining and sacrificed through submersion in 5\% N-acetyl cysteine for 10 minutes as described by Pearson, et al. Tissues were then fixed in either $2 \%$ or $4 \%$ paraformaldehyde for 2 hours prior to permeabilization with phosphate buffered saline (PBS) containing $0.05 \%$ Triton X-100. A blocking solution of 5\% non-fat dry milk and $0.05 \%$ Triton X-100 in PBS was used to reduce non-specific binding during subsequent antigen detection steps. Samples were suspended in our primary antibody cocktail containing a polyclonal immunoglobulin (rabbit anti-MET) 
specific for the MET receptor prepared in PBS (1:500), for 24 hours at $4^{\circ} \mathrm{C}$. After several washes with PBS, some samples were treated with a secondary antibody (goat anti-rabbit, 1:1000) conjugated to fluorescein isothiocyanate (FITC) for 3 hours at room temperature, while others were stored in PBS at $4^{\circ} \mathrm{C}$ (secondary omission). Planaria were subjected to a final series of PBS washes before mounting. Images were captured using a Nikon PCM 2000 confocal microscope. Our preliminary findings suggest that D. dorotocephala do not express the MET receptor under normal physiological conditions; transcription of MET following injury is being investigated.

\section{References}

[1] B.M. Carlson in "Principles of regenerative biology", ed. B.M. Carlson, (Amsterdam/Elsevier, Boston) p 1-70.

[2] J. Chmielowiec, et al., J Cell Biol 177 (2007) p.151-162.

[3] A. Fagotti, et al., Invetebrate Survival Journal 3 (2006) p.118-124.

[4] E. Gohda, et al., J Clin Invest 81 (1988) p.414-419.

[5] G.C. Gurtner, et al., Nature 453 (2008) p.314-321.

[6] T. Ishikawa, et al., Hepatology 55 (2012) p.1215-1226.

[7] S. Nakamura, et al., Endocrinol Jpn 31 (1984a) p.675-679.

[8] T. Nakamura, et al., Biochem Biophys Res Commun 122 (1984b) p.1450-1459.

[9] T. Nakamura, et al., FEBS Lett 224 (1987) p.311-316.

[10] T. Nakamura, et al., J Gastroenterol Hepatol 26 (2011) Suppl 1 p.188-202.

[11] N.J. Ovido, et al., Dis Model Mech 1 (2008) p.131-143.

[12] R. Pascolini, et al., Tissue Cell 20 (1988) p.157-163.

[13] R. Pascolini, et al., Cell Tissue Res 236 (1984) p.345-349.

[14] R. Zarnegar and G. Michalopoulos, Cancer Res 49 (1989) p.3314-3320.

[15] B. Pearson, et al., Dev Dynamics 238 (2009) p. 443-450. 